\title{
REPLANTEANDO EL DEBATE SOBRE MIGRACIONES INTERNACIONALES Y DESARROLLO: NUEVAS DIRECCIONES Y EVIDENCIAS
}

\author{
Rethinking the debate on migration and development: \\ new evidence and directions
}

María Del Carmen Villarreal Villamar

\begin{abstract}
Resumen. Este trabajo tiene como objetivo analizar los vínculos entre migraciones internacionales y desarrollo para dar cuenta de la complejidad del fenómeno y de sus nuevos rasgos. Para ello, a través de la revisión de la literatura especializada, serán examinadas las corrientes migratorias menos contempladas por los estudios sobre el tema (Sur-Sur, Norte-Sur y Norte-Norte), así como fenómenos con importancia creciente como el refugio, el cambio climático, el tránsito de personas y la influencia de los desplazamientos sobre la felicidad y el bienestar de los migrantes.
\end{abstract}

Palabras clave: migraciones internacionales, desarrollo, flujos Sur-Sur, Norte-Sur y Norte-Norte.

Abstract. This paper aims to assess the links between international migration
and development, taking in to account the complexity of this phenomenon
and its new features. In reviewing specialized literature, understudied
migratory flows such us South-South, North-South and North-North
migration will be analysed. Phenomenas with increased importance such us
refuge, climate change, transit migration and the influence of displacement
upon happiness and well-being of migrants will also be considered. Keywords: international migrations, development, South-South, NorthSouth and North-North flows.

\section{Introducción}

Históricamente los impactos que las migraciones provocan sobre el desarrollo han sido objeto de interés por parte de estudiosos y formuladores de políticas públicas. En términos generales dos son los enfoques que vinculan estas variables: optimistas y pesimistas. Su adopción está estrechamente vinculada a

\footnotetext{
* Universidade Estadual do Norte Fluminense Darcy Ribeiro. Rio de Janeiro, RJ, Brasil.
} 
la prevalencia de análisis basados en las teorías convencionales del desarrollo en el primer caso y en las teorías críticas en el segundo. Así, por ejemplo, en el siglo XIX y hasta comienzos del siglo XX los desplazamientos se consideraban, desde una perspectiva esencialmente económica, como un elemento benéfico para las zonas de expulsión y atracción. Tras la Segunda Guerra Mundial y hasta la década de los setenta, este enfoque se mantuvo asentado en las creencias desarrollistas y modernizadoras que consideraban la emigración desde zonas en desarrollo hacia sociedades más avanzadas, o el retorno desde estas últimas, como fuente de recursos económicos y transferencia de conocimientos y tecnología. Posteriormente, la crítica neo-marxista y los análisis históricos estructurales permitieron subrayar las pérdidas y la perpetuación de las asimetrías para los países emisores, en virtud de factores como la salida de población cualificada o la dependencia de las remesas. Empero, los análisis derivados de ambos abordajes facilitaron que desde los años noventa surgieran posturas pluralistas que reconocen al mismo tiempo las pérdidas y los beneficios generados por los desplazamientos ${ }^{1}$.

La importancia que ha asumido el fenómeno en las últimas décadas ha dado lugar a una gran cantidad de foros internacionales, así como a observatorios y publicaciones que lo analizan regularmente ${ }^{2}$. Sin embargo, a nivel teórico las opiniones distan de ser uniformes. En consecuencia, en los próximos apartados serán expuestos los principales rasgos de las posturas sobre migraciones internacionales y desarrollo para, sucesivamente, analizar elementos emergentes sobre la movilidad humana que sugieren la necesidad de profundizar la discusión.

\section{Debates clásicos: miradas optimistas y pesimistas}

La idea que las migraciones son un fenómeno positivo para el desarrollo es el enfoque mayoritario y uno de los principales argumentos de los organismos internacionales para incluirlas en la planificación de las agendas de desarrollo. Desde este prisma, el desarrollo no se entiende únicamente en sentido economicista como un proceso generador de riqueza y crecimiento económico, sino que, tras las críticas y reformulaciones de las que ha sido objeto este concepto, que han permitido la consideración de diferencias entre zonas geográficas o de aspectos individuales, de género o medioambientales ${ }^{3}$, se concibe como un proceso generador de bienestar social, político, económico y cultural, donde destacan el protagonismo y la capacidad de agencia de los

1 DE HASS, Hein. Migration and Development. A Theoretical Perspective.

2 Entre los principales foros cabe destacar el Diálogo de Alto Nivel sobre Migración y Desarrollo (HLD), el Foro Global sobre Migración y Desarrollo (FGMD) y la Iniciativa Conjunta sobre Migración y Desarrollo (JMDI).

3 PAINE, Anthony, PHILIPS, Nicola. Desarrollo, p. 147-148. 
individuos. Así, los desplazamientos son vistos como catalizadores de progreso que generan cadenas capaces de irradiar los beneficios obtenidos por los migrantes (económicos, sociales, políticos o culturales) hacia las comunidades y de estas al ámbito nacional ${ }^{4}$.

Para los optimistas, la emigración puede aliviar las economías de los países de origen reduciendo sus niveles de desempleo y subempleo, mientras que las remesas económicas, que se han convertido en el "nuevo mantra del desarrollo" los años noventa, estas han superado en muchos países la Ayuda Oficial al Desarrollo $(\mathrm{AOD})^{6}$, constituyendo una fuente de ingresos más constante que otros flujos de capital privado o de las Inversiones Extranjeras Directas (IED) (véase gráfico I).

\section{GRÁFICO I. Remesas (en miles de millones de dólares estadounidenses) hacia países en desarrollo en comparación con otros flujos de financiación externa (1990-2014)}

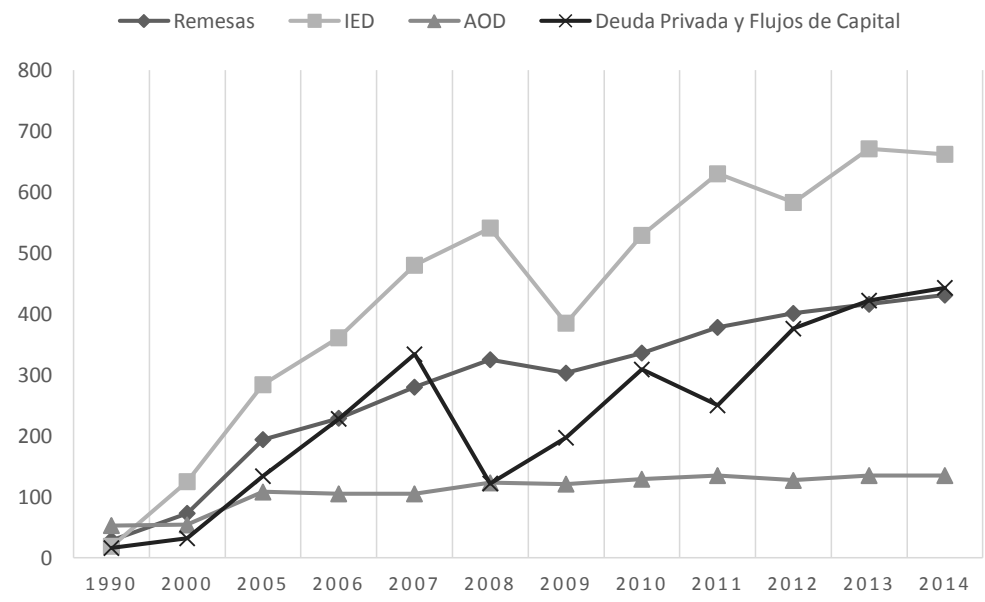

Elaboración propia. Fuente: RATHA, Dilip et alii. Migration and Remittances Factbook 2016, p. 34.

4 OIM. El bienestar de los migrantes y el desarrollo.

KAPUR, Devesh. Remittances: The New Development Mantra?, p. 7.

6 Según estimaciones del Banco Mundial, el flujo total de remesas en 2015 superó los 601 mil millones de dólares. De esta cifra, alrededor de 441 mil millones de dólares se dirigieron a países en desarrollo, lo que representa casi tres veces la ayuda oficial al desarrollo. La importancia de estos flujos aumenta, sin embargo, si se considera que el importe real es mayor pero desconocido, puesto que una parte significativa de las remesas mundiales es enviada por canales informales. En 2015, los cinco principales perceptores de remesas fueron la India, China, Filipinas, México y Francia. Con todo, para algunos países en desarrollo las remesas constituyen una parte fundamental del Producto Interno Bruto (PIB). En 2015, por ejemplo, las remesas representaron más del 25\% del PIB en Kirguistán, Tayikistán, Nepal, Tonga y Moldavia (RATHA, Dilip et alii. Migration and Remittances Factbook 2016, p. v-vi). 
Amparándose en estas consideraciones, sus sostenedores señalan que las remesas constituyen una fuente de ingresos directa que incrementa el capital de los hogares receptores, contribuye a cubrir sus necesidades básicas y puede disminuir la pobreza y la desigualdad social, mejorando los niveles de alimentación, salud, vivienda, educación, y muchas veces el acceso al crédito de los beneficiarios ${ }^{7}$. Además, si generan empleos y proyectos de desarrollo local, sus beneficios se extienden a nivel comunitario y nacional.

A nivel macroeconómico, aquellos países que reciben flujos significativos de remesas y donde las mismas representan una parte importante del PIB nacional, pueden también beneficiarse de una serie de efectos positivos, tales como el aumento de las reservas de divisas o un mayor equilibrio macroeconómico. Estos rubros adicionales pueden tener también un efecto amortiguador frente a crisis económicas, desastres naturales y conflictos civiles $^{8}$.

De forma semejante, la migración de retorno puede beneficiar a los países de origen a través de los recursos, habilidades y contactos adquiridos por los migrantes en el extranjero ${ }^{9}$. Las ventajas aumentan cuando se trata de migración cualificada que supone una ganancia de cerebros (brain gain). En consecuencia, la movilidad de personas cualificadas es vista como un fenómeno positivo y deseable para formar, por ejemplo, a las clases dirigentes de los países del Sur y se cree que sus posibles desventajas son compensadas por las remesas y toda la ayuda que los migrantes pueden brindar de forma permanente o temporal a sus países de origen.

Las diásporas ${ }^{10}$ pueden también cumplir un papel esencial en la promoción del desarrollo. En efecto, al igual que en los casos de Israel, India o Corea del Sur, a través de las remesas, de contribuciones filantrópicas privadas o colectivas, o de proyectos de inversión privada, las diásporas pueden estimular, por ejemplo, tanto el turismo como las inversiones extranjeras. Pueden desempeñar, asimismo, un papel fundamental en el crecimiento económico, en la difusión de nuevas tecnologías y promoción de la innovación ${ }^{11}$, en la diversificación

\footnotetext{
GHOSH, Bimal. Las remesas de migrantes y el desarrollo: mitos, retórica y realidades.

Ibidem; RATHA et alii, op. cit.

9 CASSARINO, Jean-Pierre. Return migration and development: the significance of migration cycles.

${ }^{10}$ Aunque el término diáspora se asocie tradicionalmente con poblaciones forzadas a salir de sus territorios ancestrales como los judíos o los armenios y aún no exista un consenso general acerca de si todas las comunidades migrantes son diásporas lo cierto es que, desde visiones no esencialistas, características como las redes y el contacto permanente que los grupos de migrantes originarios de un mismo territorio mantienen entre sí y su país de origen, el interés y solidaridad que manifiestan hacia la suerte del mismo, y principalmente elementos como su autodefinición e identificación a partir de una identidad colectiva y el cultivo de esta, nos permiten definirlos como diásporas (SHEFFER, Gabriel. Diaspora Politics: At Home Abroad, p. 9-10).

${ }^{11}$ OIM, El bienestar..., op. cit.
} 
productiva o en la reducción de la pobreza de sus países de proveniencia ${ }^{12}$, mientras que, en ámbito político, pueden fomentar la democratización de sus sociedades de origen.

Finalmente, la visión optimista se apoya en la propuesta de codesarrollo. Dicha fórmula, desarrollada en la segunda mitad de los años noventa por Sami Naïr, enfatiza las ventajas mutuas que los desplazamientos suponen en términos económicos, sociales y culturales. No obstante, su importancia radica en que otorga a los migrantes el protagonismo en todo el proceso y busca promover activamente su involucramiento en la promoción de proyectos de mejoría de las condiciones sociales y económicas de sus localidades de origen y destino ${ }^{13}$.

A contramano de estas creencias, los pesimistas consideran que los flujos actuales benefician sobre todo a las sociedades de acogida, quienes reciben gratuitamente a trabajadores jóvenes y educados fuera de su territorio que alimentan sus mercados de trabajo y contribuyen a mantener sus sistemas de previsión social. Todo esto ocurre a expensas de los países de origen que se ven afectados por el despoblamiento o envejecimiento de su población, así como por la pérdida de mano de obra o el abandono de actividades productivas. En tales condiciones, afirmar que las migraciones pueden ser fuente de desarrollo para las sociedades de origen es negativo, puesto que se delega en los migrantes las responsabilidades estatales y se les exige cumplir con compromisos que a menudo no quieren ni pueden asumir ${ }^{14}$.

En relación a las remesas, los pesimistas ponen en entredicho sus ventajas a la hora de generar procesos de desarrollo de largo aliento. Por ello, más que un recurso sostenible, son consideradas como una solución parcial y de corto plazo a problemas individuales y familiares, con escasa incidencia en el equilibrio socioeconómico y en el desarrollo de los países que las reciben ${ }^{15}$. Además de estos elementos, se enfatizan las consecuencias negativas de las remesas, entre las que sobresalen la promoción del consumo en detrimento de las actividades productivas; la mayor dependencia y vulnerabilidad de las economías receptoras; el fomento de una mentalidad rentista y la creación de un escenario en que el Estado puede verse estimulado a retraer sus funciones ante problemas que afectan a la población. Otros resultados no deseados son también la desestabilización que pueden provocar las remesas en las economías

\footnotetext{
$\overline{12}$ AGUNIAS, Dovelyn, NEWLAND, Kathleen. Hoja de Ruta para la participación de las diásporas en el desarrollo.

${ }^{13}$ GIMÉNEZ, Carlos. A favor del codesarrollo. Una llamada al diálogo y a la cooperación, p. 14.

${ }^{14}$ CASTLES, Stephen, DELGADO, Raúl. Migración y desarrollo: Perspectivas desde el Sur; DELGADO, Raúl, MÁRQUEZ, Humberto, RODRÍGUEZ, Héctor. Seis tesis para desmitificar el nexo entre migración y desarrollo.

15 Ibidem.
} 
receptoras debido a que, en gran medida, su envío utiliza medios informales que pueden facilitar la financiación de guerras o de actividades ilícitas ${ }^{16}$.

Respecto a la emigración de población cualificada, los pesimistas consideran que esta constituye una pérdida cuantiosa para el país emisor y una transferencia neta para los países receptores ${ }^{17}$. Así, partiendo de una mirada nacionalista, la población cualificada es vista como un recurso necesario para impulsar procesos de innovación, crecimiento económico y redistribución social.

Ahora bien, las repercusiones de la salida de este colectivo, definida muchas veces como pérdida o drenaje de cerebros (brain drain), no se circunscriben al sector económico, sino que afectan a diversos ámbitos esenciales para el desarrollo de un país como la salud y la educación ${ }^{18}$. Por otro lado, esta emigración priva a los países de origen de un conjunto de personas cultas y críticas, necesarias para impulsar procesos de democratización, promoción de derechos y construcción de ciudadanía. Además, los efectos negativos de la fuga de capital humano se extienden también a las sociedades anfitrionas cuando la población inmigrante ocupa empleos que no están a la altura de su formación y donde se verifica con frecuencia el fenómeno de desperdicio de talentos, también conocido como brain waste. Así, como demuestra la experiencia promovida por países latinoamericanos, y no sólo, desde este prisma la solución radica en crear iniciativas de regulación y control de estos flujos, además de establecer incentivos al retorno voluntario o programas específicos de repatriación ${ }^{19}$.

En relación a los impactos de las diásporas, se enfatizan sus límites temporales y espaciales, puesto que los lazos e intereses de los migrantes y sus descendientes por sus países natales no son eternos y, por lo general, más que con el país en su conjunto, se refieren a localidades específicas ${ }^{20}$. Por otro lado, ya que no todas las diásporas cuentan con los mismos recursos, solo

$\overline{{ }^{16} \text { KAPUR, op. cit., p. } 8 .}$

17 DELGADO et alii, op. cit.

${ }^{18}$ Los países donde existe fuga de cerebros son aquellos donde la emigración supone la salida de más del $10 \%$ de sus titulados superiores. Las zonas más sensibles a este fenómeno son en la actualidad África y el Caribe, donde se registran graves consecuencias debido a que el éxodo de profesionales involucra a áreas fundamentales para el desarrollo, tales como la salud y la educación. La gravedad de esta situación aumenta en el caso de países pequeños como Guyana, Haití, Barbados o Trinidad y Tabago que tienen a más del $60 \%$ de su personal cualificado viviendo fuera de las fronteras nacionales (RATHA et alii, op. cit.).

${ }^{19}$ VILLARREAL, María. Políticas migratórias, Transnacionalismo e desenvolvimento: o caso equatoriano.

${ }^{20}$ En el caso de la India, por ejemplo, las aportaciones de la diáspora se han concentrado especialmente en dos estados: Kerala y Guyarat, dos territorios con fuerte emigración, además de ser pioneros junto con el estado de Punjab en la creación de organismos de apoyo a los miembros de la diáspora y en el establecimiento de vínculos y medidas para potenciar sus contribuciones (AGUNIAS, NEWLAND, op. cit.). 
algunas pueden cumplir un papel relevante en el desarrollo de sus territorios natales. Por último, se cree que estos grupos pueden afectar negativamente a sus sociedades apoyando crisis o conflictos locales.

A su vez, los críticos del codesarrollo denuncian que esta estrategia más que ayudar a los países de origen, busca controlar los flujos migratorios y promover el retorno de los inmigrantes no deseados. Otros de sus límites son su aún escasa definición, así como el haber sido concebida y financiada por los países desarrollados, lo que provoca normalmente la primacía de los intereses de estos Estados, por encima de los objetivos de los territorios locales y de las organizaciones de migrantes ${ }^{21}$.

Por fin, los pesimistas indican los limitados efectos de la migración de retorno, resaltando que esta depende de una serie de factores como el tipo de regreso y el contexto en el que se produce, las condiciones económicas, sociales y políticas del Estado de origen, así como de las características y el volumen de la población retornada. Al contrario del mito sobre el emigrante exitoso que vuelve con formación, recursos y una mentalidad emprendedora o propensa al desarrollo económico -en línea con presupuestos modernizadores y desarrollistas-, muchos procesos se verifican de manera forzada o semiforzada ${ }^{22}$, al igual que por factores ajenos respecto a la contribución al desarrollo, tales como la jubilación de los migrantes, la nostalgia de casa, motivos familiares o de salud. En este sentido, no es posible afirmar que todos los retornados deseen o tengan la capacidad de aportar al desarrollo de sus sociedades de origen.

\section{Nuevos elementos y agendas de investigación}

En un momento en el que, de acuerdo a las estadísticas del Banco Mundial, existen más de 247 millones de migrantes internacionales que representan el 3,4\% de la población global ${ }^{23}$, comprender plenamente las múltiples interacciones existentes entre el binomio migraciones y desarrollo requiere considerar todos los elementos hasta ahora enunciados, así como ciertas tendencias que emergen del panorama actual. De hecho, en los análisis sobre el desplazamiento humano ha prevalecido el estudio de los flujos Sur-Norte, mientras que el resto de las direcciones (Sur-Sur, Norte-Sur y Norte-Norte), a pesar de su importancia numérica y de los desafíos globales que suponen, han sido relativamente menos estudiadas (ver gráfico II).

\footnotetext{
${ }^{21}$ GIMÉNEZ, op. cit., p. 15.

${ }^{22}$ CASSARINO, op. cit.

${ }^{23}$ RATHA et alii, op. cit.
} 


\section{GRÁFICO II. Millones de migrantes internacionales en las cuatro direcciones de la migración según el Banco Mundial (BM), el PNUD y el Departamento de Asuntos Económicos de las Naciones Unidas (DAES) (2010) ${ }^{24}$}

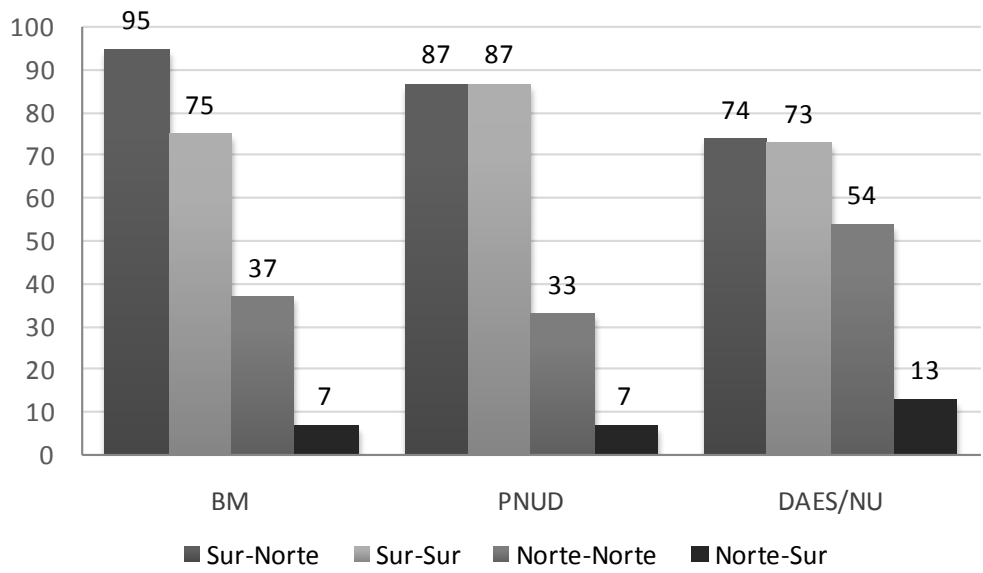

Elaboración propia. Fuente: OIM, El bienestar..., op. cit., p. 57

Más allá de los efectos de la migración de los países en desarrollo hacia las regiones más ricas del planeta, como sostienen diversos estudios ${ }^{25}$, la geografía de los flujos migratorios está cambiando y es crucial pensar en los impactos contradictorios generados por las corrientes Sur-Sur, así como en los heterogéneos resultados a los que pueden dar lugar los desplazamientos Norte-Sur o Norte-Norte, en un contexto en que el aumento de la movilidad humana obedece a transformaciones sociales, económicas, demográficas y ambientales de carácter mundial. En el caso de la migración Sur-Sur, por ejemplo, aunque existen divergencias sobre su dimensión real, de acuerdo al Banco Mundial, equivale al 38\% del contingente de migración global ${ }^{26}$ y supera los flujos Sur-Norte que suponen alrededor del $34 \%{ }^{27}$. En efecto, más de la mitad de los 20 corredores migratorios del mundo se encuentra a lo largo

\footnotetext{
${ }^{24}$ Las diferencias en los cálculos sobre cada dirección son resultado de los diversos criterios utilizados por las agencias internacionales para clasificar a los países como miembros del Sur o del Norte del mundo.

${ }^{25}$ OIM, El bienestar..., op. cit.; OIM. Los migrantes y las ciudades: nuevas colaboraciones para gestionar la movilidad; RATHA et alii, op. cit.; WIHTOL DE WENDEN, Catherine. Las nuevas migraciones.

${ }^{26}$ En términos regionales las proporciones de estos flujos pueden ser aún más significativas. Este es el caso de África y Asia, donde la migración intrarregional es muy superior a los flujos en dirección a Europa u otras zonas y supera el 50\% del total (MELDE, Susanne et alii. The SouthSouth Migration and Development Nexus, p. 8).

${ }^{27}$ RATHA et alii, op. cit., p. v.
} 
del eje Sur-Sur y un significativo porcentaje de estos desplazamientos tiene carácter intrarregional, o se asocia con espacios migratorios regionales ${ }^{28}$.

Las razones que alientan este tipo de desplazamientos son múltiples, pero en términos generales sobresalen las desigualdades entre países y los mejores indicadores económicos de las sociedades de destino, la estabilidad política, la paz y las mejores oportunidades que ofrecen en diferentes esferas, así como la existencia de redes y modelos de integración regional que facilitan el cruce de fronteras ${ }^{29}$. Otros factores que alimentan o influencian las migraciones en dirección Sur-Sur, son también el crecimiento de la población, así como el cambio climático, provocado por el calentamiento global y la degradación ambiental.

Se calcula que entre 200 millones y 1.000 millones de personas serán desplazadas en los próximos cuarenta años a consecuencia del cambio climático -en combinación con otros factores-, y que la mayor parte de estos migrantes será originaria del Sur del mundo ${ }^{30}$. Los efectos de esta problemática son y serán especialmente evidentes respecto al acceso al agua, la seguridad alimentaria y la salud, contribuyendo a exacerbar las causas de conflictos y crisis locales ${ }^{31}$. Empero, para hacer frente a estos desafíos, los países del Sur cuentan con políticas y mecanismos de prevención o enfrentamiento de emergencias humanitarias y climáticas insuficientes y usualmente más débiles que los de los países ricos.

En lo que concierne al perfil de los migrantes en el eje Sur-Sur, a pesar de que existe una proporción significativa de personas cualificadas ${ }^{32}$, ya que estos desplazamientos se producen mayoritariamente entre países vecinos o con relativas facilidades económicas y logísticas, un gran número de personas posee escasa instrucción y accede al mercado laboral para desempeñar funciones de media y, prevalentemente, baja calificación ${ }^{33}$. El ingreso de migrantes con estas características, o por lo menos su aumento, más allá de las razones antes expuestas, es también resultado de las cada vez más restrictivas y selectivas políticas migratorias en los países del Norte. De esta manera, porcentajes significativos de la migración del circuito Sur-Sur son, en parte, formados por la población "no bienvenida" o no seleccionada por los países desarrollados

\footnotetext{
${ }^{28}$ WIHTOL DE WENDEN, op. cit.

${ }^{29}$ RATHA, Dilip, SHAW, William. South-South Migration and Remittances.

${ }^{30}$ LACZKO, Frank, PIGUET, Etienne. Regional perspectives on migration, the environment and climate change.

${ }^{31}$ MELDE et alii, op. cit.

${ }^{32}$ Aunque existen desplazamientos espontáneos en esta dirección, cabe también mencionar los programas de atracción de talentos o las facilidades brindadas para migrantes cualificados por parte de países como Sudáfrica, Malasia o los países del Golfo Pérsico.

33 RATHA, SHAW, op. cit.; MELDE et alii, op. cit.
} 
y con frecuencia constituyen una forma de migración de los pobres o menos favorecidos en búsqueda de alternativas de sobrevivencia.

Otras características de la migración Sur-Sur son su carácter mayoritariamente temporal y circular, sujeto a contratos y con elevadas tasas de retorno, así como sus mayores niveles de informalidad, lo que determina que sus protagonistas sean más irregulares que en el resto de los circuitos ${ }^{34}$. En razón de estas variables, una vez en los países de arribo -en tanto países de tránsito o destino final- la desprotección y abusos sobre los inmigrantes son generalizados, no solo por sus condiciones de llegada, sino también por la frecuente concentración en las periferias de las zonas urbanas carentes de servicios básicos ${ }^{35}$, así como por las características de las sociedades locales que presentan elevados índices de desempleo, informalidad y dificultades de acceso a servicios y derechos. Aquí también vale destacar la vigencia de políticas migratorias restrictivas, una legislación poco eficaz y escasos instrumentos para garantizar el cumplimiento de los derechos de los migrantes, facilitar su integración y evitar que los mismos sean víctimas de racismo o formas de exclusión.

Una problemática no menos central en el caso de las migraciones Sur-Sur es el escaso conocimiento que se tiene sobre la misma, en virtud de la falta de información y estadísticas completas sobre el fenómeno. De esta suerte, los impactos de naturaleza positiva o negativa sobre las sociedades receptoras aún son poco comprendidos.

A su vez, las consecuencias sobre las sociedades de partida necesitan mayores estudios, pues sin duda estas migraciones presentan potencialidades para el desarrollo. No obstante, aunque los datos varíen entre regiones y países $^{36}$, la realidad muestra que los migrantes de este eje envían menos remesas a casa, en parte por la precariedad y bajos ingresos que perciben $y$, en parte, también por las bajas diferencias monetarias y salariales que existen entre los países del Sur. Otra razón que contribuye a esta problemática es que muchas veces las remesas son enviadas por canales informales, lo que determina que su importe real y el peso que ejercen sobre la economía local sean desconocidos.

\footnotetext{
34 Ibidem.

${ }^{35}$ En tales circunstancias con frecuencia los migrantes deben hacer frente también a la violencia urbana y a diversas formas de degradación medioambiental que amenazan su salud y su seguridad alimentaria (OIM, Los migrantes..., op. cit.).

${ }^{36}$ En África, por ejemplo, las remesas intrarregionales son mayores que en Asia o en América Latina. A nivel nacional, por otro lado, las remesas enviadas por medios informales pueden adquirir dimensiones muy elevadas como en Lesoto, donde constituyen el $87 \%$ de los flujos enviados (NALANCE et alii. 2012, apud MELDE et alii, op. cit., p. 12).
} 
Las características apenas descritas determinan que los migrantes en la dirección Sur-Sur sean los menos optimistas en relación a sus proyectos migratorios y consideren difícil alcanzar un nivel de vida satisfactorio ${ }^{37}$. Estas declaraciones son resultado de una reciente encuesta mundial Gallup ${ }^{38}$ sobre el bienestar de los migrantes. Los datos muestran que, en el circuito Sur-Sur, los migrantes señalan como determinantes de sus bajos niveles de bienestar las dificultades que enfrentan para integrarse en el mercado laboral formal, con los consecuentes obstáculos de acceso a derechos y servicios sociales; los problemas de seguridad que afligen a las sociedades de acogida; los bajos niveles de confianza en las instituciones y autoridades locales e insuficientes garantías de estabilidad económica. Como resultado, los migrantes de este eje son los menos felices en términos materiales y se demuestran pesimistas frente a su vida presente y futura, al tiempo que evalúan su trayectoria de forma negativa frente a personas con condiciones semejantes en las sociedades de origen, afirmando que su situación actual es peor de la que tendrían de no haber emigrado.

Elementos que merecen también mayor atención son el refugio y el tránsito de personas, especialmente en el circuito Sur-Sur. En efecto, en los últimos años guerras, violencia generalizada, heterogéneas formas de discriminación y persecución, así como innumerables violaciones a los derechos humanos determinaron un crecimiento sin precedentes del número de refugiados, desplazados internos y solicitantes de refugio, equivalente a 65,3 millones de personas ${ }^{39}$. A pesar de su importancia numérica, el 86\% de los refugiados son acogidos por países en desarrollo, entre los que destacan Turquía, Pakistán, Líbano, la República Islámica de Irán, Etiopía y Jordania. Este elemento, aunado a hechos como que el 51\% de la población refugiada es menor de edad y constituye un grupo de alta vulnerabilidad ${ }^{40}$, determinan que tanto los recursos económicos como las capacidades de estos Estados se vean sometidos a altos niveles de presión. Con diversos problemas estructurales, a menudo estos no poseen ni condiciones ni políticas adecuadas

\footnotetext{
${ }^{37}$ OIM, El bienestar..., op. cit.

${ }^{38}$ Los datos corresponden al periodo 2009-2011 y son fruto de una encuesta en la que participaron alrededor de medio millón de adultos, siendo aproximadamente 25.000 migrantes de primera generación y 441.000 residentes nacidos en el país. Las consideraciones personales sobre el proceso migratorio de los participantes en las cuatro direcciones derivan esencialmente de las condiciones en las que se ha producido el hecho migratorio, así como de su formación, estatus legal, condiciones en el país de recepción y comparación de sus condiciones de vida con las de aquellos que permanecieron en los contextos de salida (Ibidem, p. 109).

39 En esta cifra se incluyen 21,3 millones de personas refugiadas (16,1 millones bajo mandato del ACNUR y 5,2 millones de refugiados palestinos registrados por el UNRWA, 40,8 millones de personas desplazadas internas y 3,2 millones de solicitantes de asilo (ACNUR. Tendencias Globales. Desplazamiento Forzado en 2015, p. 2).

${ }^{40}$ Ibidem, p. 52.
} 
para brindar una correcta recepción, protección o asistencia y mucho menos para garantizar la plena integración de este colectivo.

La situación de tránsito en la que viven millones de migrantes, especialmente en los flujos Sur-Sur ${ }^{41}$, es otro fenómeno digno de nota. Aunque no existe una definición universalmente válida y aceptada, el tránsito describe, por lo general, la migración de personas -en condiciones regulares o no- y por un tiempo indefinido hacia un país, con la intención de dirigirse y vivir en otro lugar, bien sea por los beneficios que un tercer país ofrece, por las características y facilidades brindadas por el país de tránsito, o en razón de políticas migratorias restrictivas que las obligan a permanecer en un lugar diferente al de su destino, a la espera de condiciones mejores para poder emigrar nuevamente. No obstante, si bien el concepto de tránsito describe la situación intermedia entre la emigración y el establecimiento en un determinado territorio ${ }^{42}$, otras voces aseveran que es una categoría política, que engloba situaciones disímiles y que sirve para externalizar y reforzar los controles migratorios como en el caso europeo ${ }^{43}$.

Como demuestran las experiencias de México y Marruecos, el tránsito puede durar largos periodos o convertirse en definitivo ${ }^{44}$, con importantes repercusiones sobre la seguridad y la protección de los migrantes. En estas circunstancias, gracias a la falta de perspectivas e indefinición de sus estatus, estos acaban integrándose en el mercado laboral en condiciones precarias o en actividades irregulares. Con todo, pese a que en estas condiciones es posible que los migrantes trabajen y algunas veces envíen remesas a sus comunidades de origen, cabe mencionar también los altos riesgos de sufrir violaciones a los derechos humanos, particularmente cuando se trata de mujeres y menores de edad. Ambos colectivos, al igual que otros con características concretas, exigen que se distingan claramente las necesidades de cada grupo y los desafíos que suponen para las políticas de los Estados receptores.

Por su parte, en base a las diversas clasificaciones del PNUD, del Banco Mundial y del DAES ${ }^{45}$, la migración Norte-Sur, representa entre el 3 y el $6 \%$ de la migración mundial, involucrando entre 7 y 13 millones de migrantes, pero la evidencia muestra que está en aumento. No obstante, estos desplazamientos no afectan al Sur en su conjunto, sino que se concentran en algunos países

${ }^{41}$ OIM, Los migrantes..., op. cit.

${ }^{42}$ PAPADOPOULOU, Aspasia. Transit migration. The missing link between emigration and settlement, p. 3.

${ }^{43}$ DÜVELL, Franck. Transit Migration: A Blurred and Politicised Concept.

${ }^{44}$ PAPADOPOULOU, op. cit.; DÜVELL, op. cit.; OIM, Los migrantes..., op. cit.

45 Para el Banco Mundial y el PNUD, esta corriente representa el 3\% de la migración mundial, mientras que en base a la clasificación del DAES supone el 6\% (OIM, El bienestar..., op. cit., p. 57). 
como China, Brasil, India, Nigeria o Malasia y, dentro de estos, en las zonas más desarrolladas ${ }^{46}$.

Las razones que estimulan esta movilidad son complejas e incluyen la mejoría de las condiciones económicas, políticas y sociales de los países del Sur; la transferencia y expansión de las empresas transnacionales en los países en desarrollo; las consecuencias de la crisis global en los países del Norte que ha provocado la búsqueda de alternativas de empleo para sus ciudadanos y el retorno de numerosos inmigrantes hacia sus países de origen; la creciente demanda de mano de obra cualificada y la oferta de salarios atractivos; la mejor oferta formativa y precios más accesibles que los países del Norte en el caso de la migración estudianti ${ }^{47}$ y, por último, condiciones más favorables, así como un clima más agradable para retirarse y transcurrir los años posteriores a la jubilación ${ }^{48}$. Otros factores que alientan estos desplazamientos son las relaciones históricas, de carácter colonial o no, entre países y los vínculos amplios que se establecen entre naciones a consecuencia de acuerdos económicos o políticos, así como las motivaciones de carácter familiar, de género o ideológicas que motivan, por ejemplo, la llegada de activistas o defensores de derechos humanos ${ }^{49}$.

A la hora de comprender las dimensiones e impactos de estos flujos existen problemas de fiabilidad de datos debido a que muchas veces son incompletos o incomparables e incluyen a menudo a población retornada o a sus descendientes. A pesar de ello, las implicaciones para el desarrollo en los países de origen y destino ameritan atención creciente. Para los países del Norte las principales ventajas de esta emigración son la menor presión sobre el mercado laboral y el aumento del flujo de remesas, aunque con impactos menores respecto al circuito Norte-Sur ${ }^{50}$. A la inversa, entre los factores negativos cabe destacar la pérdida de población y, especialmente, la fuga de cerebros, así como la disminución de los ingresos fiscales para Estados que ya adolecen de una recaudación insuficiente respecto a sus demandas de gasto social ${ }^{51}$. En el mismo orden de ideas, los países del Sur pueden verse beneficiados por la

\footnotetext{
${ }_{46}$ Ibidem; OIM, Los migrantes..., op. cit.

${ }^{47}$ Otro elemento que puede motivar el desplazamiento Norte-Sur, incluyendo el de jubilados, es también la voluntad de aumentar sus ingresos dedicándose, por ejemplo, a abrir nuevas posibilidades de negocio en ámbito turístico, gastronómico, sanitario o inmobiliario (RADITSCH, Marisa. North-South Migration: the impact of international immigration in the municipality of Chapala, Jalisco, México, p. 98).

${ }^{48}$ LACZKO, Frank, BRIAN, Tara. North-south migration: a different look at the migration and development debate; WIHTOL DE WENDEN, op. cit.

${ }^{49}$ OIM, El bienestar..., op. cit.; OIM, Los migrantes..., op. cit.; WIHTOL DE WENDEN, op. cit.

${ }^{50}$ De acuerdo a las estadísticas del Banco Mundial, en 2015 se enviaron 27,9 mil millones de remesas en dirección Sur-Norte, lo que equivale al 5\% del total de remesas internacionales (RATHA et alii, op. cit., p. 28).

${ }^{51}$ LACZKO, BRIAN, op. cit., p. 17.
} 
llegada de capital humano e inversiones, por la recuperación o la ganancia de talentos (reverse brain drain), por la promoción de sectores como el turismo, así como por la difusión de nuevas tecnologías y el establecimiento de redes y contactos que impulsen la economía y la productividad. Otros elementos importantes, por lo menos a nivel comunitario, son la difusión de obras de caridad e iniciativas de voluntariado, al igual que los proyectos de desarrollo local promovidos por los ciudadanos del Norte que pueden beneficiar a la colectividad y que, indirectamente, pueden disminuir los motivos para emigrar de estos territorios ${ }^{52}$.

Por su parte, entre las desventajas provocadas por las migraciones Norte-Sur se enumeran el aumento de la inflación y del coste de vida en lo relativo a vivienda y servicios, el aumento de la competitividad laboral y la consecuente pérdida de trabajo para los habitantes locales, así como la dependencia de los recursos de los extranjeros ${ }^{53}$. En el caso de la emigración de retorno, los impactos de signo negativo pueden deberse al regreso de población poco cualificada o que, al permanecer fuera del país por largos periodos y realizando funciones para las que se requiere baja formación, experimenta de facto una pérdida de sus habilidades.

Una consecuencia no menos nociva, es la concentración de los habitantes del Norte en pequeñas ciudades $y$, dentro de estas, en determinadas zonas o vecindarios. Dicha concentración puede dar lugar al surgimiento de islas u oasis de prosperidad artificiales dotados de servicios propios y privilegios en medio a la miseria, al tiempo que puede provocar importantes impactos sobre la cultura y la economía local, debido a, por ejemplo, la apertura de restaurantes y negocios de productos extranjeros, así como a la llegada de multinacionales y empresas que buscan satisfacer las demandas de esta población ${ }^{54}$. Como corolario, puede producirse el malestar de los nativos tanto por constituir un grupo de segunda clase como por comparar con los extranjeros sus ingresos y capacidad de consumo.

En lo relativo al bienestar de los migrantes, las corrientes procedentes del Norte en dirección al Sur y las del circuito Norte-Norte declaran resultados mayoritariamente positivos en relación al bienestar financiero, laboral, social, comunitario y físico. De hecho, a pesar de las dificultades relacionadas con el funcionamiento de las instituciones y servicios públicos o de las insuficientes garantías en materia de seguridad y transparencia, los que emigran de Norte a Sur disfrutan de beneficios como un mayor poder adquisitivo o mejores empleos, consideran que la emigración no supone una diferencia en sus

\footnotetext{
${ }_{52}$ RADITSCH, op. cit., p. 106.

53 OIM, El bienestar..., op. cit.

${ }^{54}$ RADITSCH, op. cit., p. 98.
} 
proyectos, y evalúan su vida de la misma forma que sus semejantes en los países de origen.

En el caso de las migraciones Norte-Norte las mejores oportunidades y las garantías ofrecidas por los Estados de recepción son los principales fundamentos para expresar mejores niveles de bienestar y para evaluar de manera más positiva su vida respecto a las demás corrientes, sobre todo en relación a las finanzas, disfrute de derechos y acceso a servicios públicos. La migración en este circuito supone 33 millones de personas de acuerdo al PNUD, 37 millones según el Banco Mundial y 54 millones según el DAES de las Naciones Unidas, lo que equivale respectivamente a un porcentaje de 15, 17 o $25 \%$ del contingente mundial de migrantes ${ }^{55}$. Sin embargo, pese a que en términos absolutos la movilidad humana es mayor en el Sur, las personas se desplazan más y con mayores facilidades en el Norte.

Por otra parte, al igual que en los casos antes descritos, estas corrientes obedecen a múltiples razones (económicas, sociales, políticas o culturales), pero a diferencia de las demás, se distinguen por involucrar a población con mayor cualificación y por incluir una presencia mayoritaria de mujeres y de personas en edad de trabajar, ubicadas principalmente en un rango de entre 25 y 49 años $^{56}$. Factores como estos, junto a las remesas o las posibilidades brindadas por las diásporas y la migración de retorno hacen que las potencialidades de los migrantes en esta dirección- para aumentar o mejorar el desarrollo de sus sociedades de partida- sean significativas, pero aun insuficientemente conocidas.

\section{Reflexiones finales}

En nuestro trabajo hemos visto, en primer lugar, que más allá de las visiones clásicas de carácter optimista y pesimista, son necesarios enfoques que tengan en cuenta los efectos positivos, negativos o neutros de los desplazamientos, al igual que sus consecuencias duales y a veces contradictorias, dependiendo del contexto, época, volumen, características de la población involucrada o del papel de las políticas de los Estados de origen y recepción.

En segundo lugar, aunque las investigaciones más recientes muestran que las migraciones internacionales pueden ser un vector de desarrollo, bajo determinadas circunstancias y con importantes limitaciones, también dan cuenta del elevado precio en términos de bienestar individual y familiar de los protagonistas del proceso, gracias a lo cual, las reflexiones excesivamente optimistas no tienen cabida. Por ende, si la movilidad humana es un hecho y es capaz de contribuir a impulsar procesos de desarrollo, sus contribuciones

\footnotetext{
${ }_{55}$ OIM, El bienestar..., op. cit., p. 57.

${ }^{56}$ Ibidem, p. 68.
} 
pueden ser fomentadas, pero estas no deben sustituir el rol ni mucho menos las responsabilidades de los Estados y de la comunidad internacional en la promoción del progreso global.

En tercer lugar, los flujos en dirección Sur-Sur, Norte-Sur y NorteNorte, así como sus heterogéneos impactos sobre el desarrollo hacen patente la necesidad de superar análisis binarios que dan cuenta apenas de las migraciones desde países del Sur hacia naciones desarrolladas y no expresan debidamente la complejidad del fenómeno. De hecho, las migraciones no solo se han globalizado involucrando a todas las regiones del mundo, sino que los factores que las condicionan y alimentan se han multiplicado, por lo que las mismas están destinadas a aumentar y con características siempre más diversificadas.

En síntesis, para comprender cabalmente las múltiples relaciones entre migraciones internacionales y desarrollo es necesario adoptar una mirada integral. Como hemos visto, no todo sobre los vínculos de estas dos variables está dicho. A la hora de analizar el fenómeno y diseñar políticas eficaces para encauzar las aportaciones de los migrantes hacia el desarrollo, es crucial aumentar el diálogo entre todos los actores involucrados, considerando todas las direcciones, expresiones e impactos del fenómeno, y poniendo al centro del debate las desigualdades entre regiones que son las que condicionan y alimentan la mayor parte de la movilidad humana.

\section{Bibliografía}

ACNUR (Alto Comisionado de las Naciones Unidas para los Refugiados). Tendencias Globales. Desplazamiento Forzado en 2015. Forzados a Huir. Ginebra: ACNUR, 2015.

AGUNIAS, Dovelyn; NEWLAND, Kathleen. Hoja de Ruta para la participación de las diásporas en el desarrollo. Un manual para políticos y profesionales de los países de origen y acogida. Ginebra: OIM, MPI, 2012.

CASTLES, Stephen; DELGADO, Raúl (coords.). Migración y desarrollo: Perspectivas desde el Sur. México: Universidad Autónoma de Zacatecas, 2007.

CASSARINO, Jean-Pierre. Return migration and development: the significance of migration cycles. In TRIANDAFYLLIDOU, Anna (ed.). Routledge handbook of immigration and refugee studies. Abingdon: New York, Routledge, 2016.

DE HASS, Hein. Migration and Development. A theoretical Perspective. International Migration Review, v. 44, n. 1, 2010, p. 227-264.

DELGADO, Raúl; MÁRQUEZ, Humberto; RODRÍGUEZ, Héctor. Seis tesis para desmitificar el nexo entre migración y desarrollo. Migración y Desarrollo, Zacatecas, n. 12, 2009, p. 27-52.

DÜVELL, Franck. Transit Migration: A Blurred and Politicised Concept. Population, Space and Place, v. 18, n. 4, 2012, p. 415-427. 
GHOSH, Bimal. Las remesas de migrantes y el desarrollo: mitos, retórica y realidades. Ginebra: Organización Internacional para las Migraciones (OIM), Proceso de la Haya sobre Refugiados y Migración (THP), 2006.

GIMÉNEZ, Carlos. A favor del codesarrollo. Una llamada al diálogo y a la cooperación. Prólogo. In LACOMBA, Joan; FALOMIR, Fernando (eds.). De las migraciones como problema a las migraciones como oportunidad. Madrid: La Catarata, 2010.

KAPUR, Devesh. Remittances: The New Development Mantra? G-24, Discussion Paper Series, United Nations, 2004. Disponible en: < https://ideas.repec.org/p/ unc/g24pap/29.html>. Consultado: 13.11.2016.

LACZKO, Frank; BRIAN, Tara. North-south migration: a different look at the migration and development debate. Migration Policy Practice, v. 3, n., 3, 2013, p. 14-19.

LACZKO, Frank; PIGUET, Etienne. Regional perspectives on migration, the environment and climate change. In PIGUET, Etienne; LACZKO, Frank (eds.). People on the move in a changing climate. The regional impact of environmental change on migration. Ginebra: OIM, Springer, 2014.

MELDE, Susanne; ANICH, Rudolf; CRUSH, Jonathan; OUCHO, John. Introduction: The South-South Migration and Development Nexus. In ANICH, Rudolf; CRUSH, Jonathan; MELDE, Susanne; OUCHO, John (eds.). A new perspective on human mobility in the South. Global Migration Issues 3. Ginebra: IOM, Springer, 2014.

OIM (Organización Internacional para las Migraciones). El bienestar de los migrantes y el desarrollo. Informe sobre las Migraciones en el Mundo. Ginebra: OIM, 2013. OIM. Los migrantes y las ciudades: Nuevas colaboraciones para gestionar la Movilidad. Informe sobre las Migraciones en el Mundo. Ginebra: OIM, 2015.

PAPADOPOULOU, Aspasia. Transit migration. The missing link between emigration and settlement. New York: Palgrave Macmillan, 2008.

PAINE, Anthony; PHILLIPS, Nicola. Desarrollo. Madrid: Alianza Editorial, 2012.

RADITSCH, Marisa. North-South Migration: the impact of international immigration in the municipality of Chapala, Jalisco, México. Hagira, v. 4, n. 10, 2015, p. 94111.

RATHA, Dilip; SHAW, William. South-South Migration and Remittances. Documento de Trabajo del Banco Mundial, n. 102, Washington, DC, 2007. Disponible en: <http://siteresources.worldbank.org/INTPROSPECTS/Resourc es/334934-1110315015165/SouthSouthMigrationandRemittances.pdf $>$. Consultado: 13.07.2015.

RATHA, Dilip; PLAZA, Sonia; DERVISEVIC, Ervin (comp.). Migration and Remittances Factbook 2016. Global Knowledge Partnership on Migration and Development, (KNOMAD). Washington: Banco Mundial, 2016.

SHEFFER, Gabriel. Diaspora Politics: At Home Abroad. New York: Cambridge University Press, 2003. 
VILLARREAL, María. Políticas migratórias, Transnacionalismo e desenvolvimento: o caso equatoriano. Cadernos Obmigra, v. 1, n. 3, 2015, p. 39-63.

WIHTOL DE WENDEN, Catherine. Las nuevas migraciones. Sur, v. 13, n. 23, 2016, p. 17-28.

Recibido para publicación en 31.03.2017

Aceptado para publicación en 30.10.2017

Received for publication in March 315t, 2017

Accepted for publication in October 30

ISSN impresso 1980-8585

ISSN eletrônico 2237-9843

http://dx.doi.org/10.1590/1980-85852503880005112 